Bull. Korean Math. Soc. 50 (2013), No. 4, pp. 1221-1233

http://dx.doi.org/10.4134/BKMS.2013.50.4.1221

\title{
ON SOME MODULAR EQUATIONS AND THEIR APPLICATIONS II
}

\author{
Dae Hyun Paek and Jinhee Yi
}

\begin{abstract}
We first derive some modular equations of degrees 3 and 9 and present their concise proofs based on algebraic computations. We then use these modular equations to establish explicit relations and formulas for the parameterizations for the theta functions $\varphi$ and $\psi$. In addition, we find specific values of the parameterizations to evaluate some numerical values of the cubic continued fraction.
\end{abstract}

\section{Introduction}

We begin this section by introducing Ramanujan's definition of his general theta function. For $|a b|<1$, define

$$
f(a, b):=\sum_{n=-\infty}^{\infty} a^{n(n+1) / 2} b^{n(n-1) / 2} .
$$

Note that two special cases of $f(a, b)$ are defined by, for $|q|<1$,

$$
\varphi(q):=f(q, q)=\sum_{n=-\infty}^{\infty} q^{n^{2}}=\left(-q ; q^{2}\right)_{\infty}^{2}\left(q^{2} ; q^{2}\right)_{\infty}
$$

and

$$
\psi(q):=f\left(q, q^{3}\right)=\sum_{n=0}^{\infty} q^{n(n+1) / 2}=\frac{\left(q^{2} ; q^{2}\right)_{\infty}}{\left(q ; q^{2}\right)_{\infty}}
$$

where

$$
(a ; q)_{\infty}:=\prod_{n=0}^{\infty}\left(1-a q^{n}\right)
$$

Received August 12, 2012; Revised January 11, 2013.

2010 Mathematics Subject Classification. Primary 11F27, 33C90; Secondary 11F20, $33 \mathrm{C} 05,33 \mathrm{C} 75$.

Key words and phrases. theta functions, modular equations, continued fractions.

This work was supported by the Education Research Institute, Busan National University of Education in 2013. 
Let $a, b$, and $c$ be arbitrary complex numbers except that $c$ cannot be a nonpositive integer. Then, for $|z|<1$, the Gaussian or ordinary hypergeometric function ${ }_{2} F_{1}(a, b ; c ; z)$ is defined by

$$
{ }_{2} F_{1}(a, b ; c ; z):=\sum_{n=0}^{\infty} \frac{(a)_{n}(b)_{n}}{(c)_{n} n !} z^{n},
$$

where $(a)_{0}:=1$ and $(a)_{n}:=a(a+1)(a+2) \cdots(a+n-1)$ for each positive integer $n$.

Now the complete elliptic integral of the first kind $K(k)$ is defined by

$$
K(k):=\int_{0}^{\pi / 2} \frac{d \theta}{\sqrt{1-k^{2} \sin ^{2} \theta}}=\frac{\pi}{2}{ }_{2} F_{1}\left(\frac{1}{2}, \frac{1}{2} ; 1 ; k^{2}\right)=\frac{\pi}{2} \varphi^{2}\left(e^{-\pi \frac{K^{\prime}}{K}}\right),
$$

where $0<k<1, K^{\prime}=K\left(k^{\prime}\right)$, and $k^{\prime}=\sqrt{1-k^{2}}$. The number $k$ is called the modulus of $K$ and $k^{\prime}$ is called the complementary modulus.

Let $K, K^{\prime}, L$, and $L^{\prime}$ denote complete elliptic integrals of the first kind associated with the moduli $k, k^{\prime}, l$, and $l^{\prime}$, respectively, where $0<k<1$ and $0<l<1$. Suppose that

$$
\frac{L^{\prime}}{L}=n \frac{K^{\prime}}{K}
$$

holds for some positive integer $n$. A relation between $k$ and $l$ induced by (1.2) is called a modular equation of degree $n$.

If we set

$$
q=\exp \left(-\pi \frac{K^{\prime}}{K}\right) \text { and } q^{\prime}=\exp \left(-\pi \frac{L^{\prime}}{L}\right),
$$

we see that (1.2) is equivalent to the relation $q^{n}=q^{\prime}$. Hence a modular equation can be viewed as an identity involving theta functions at the arguments $q$ and $q^{n}$.

Note that the definition of a modular equation mentioned above is the one used by Ramanujan, but we emphasize that there are several definitions of a modular equation in the literature. For example, refer the books by R. A. Rankin in [5] and B. Schoeneberg in [6] for other definitions of a modular equation. Following Ramanujan, set $\alpha=k^{2}$ and $\beta=l^{2}$, then we say that $\beta$ has degree $n$ over $\alpha$. By the relationship between complete elliptic integrals of the first kind and hypergeometric function, we have

$$
n \frac{{ }_{2} F_{1}\left(\frac{1}{2}, \frac{1}{2} ; 1 ; 1-\alpha\right)}{{ }_{2} F_{1}\left(\frac{1}{2}, \frac{1}{2} ; 1 ; \alpha\right)}=\frac{{ }_{2} F_{1}\left(\frac{1}{2}, \frac{1}{2} ; 1 ; 1-\beta\right)}{{ }_{2} F_{1}\left(\frac{1}{2}, \frac{1}{2} ; 1 ; \beta\right)} .
$$

Let $z_{n}=\varphi^{2}\left(q^{n}\right)$. Then the multiplier $m$ for degree $n$ is defined by

$$
m:=\frac{\varphi^{2}(q)}{\varphi^{2}\left(q^{n}\right)}=\frac{z_{1}}{z_{n}} .
$$

Next we introduce the definitions of 4 parameterizations for the theta functions $\varphi$ and $\psi$ from $[7,8,10]$. For any positive real numbers $k$ and $n$, define 
$h_{k, n}$ by

$$
h_{k, n}:=\frac{\varphi(q)}{k^{1 / 4} \varphi\left(q^{k}\right)},
$$

where $q=e^{-\pi \sqrt{n / k}}$, define $h_{k, n}^{\prime}$ by

$$
h_{k, n}^{\prime}:=\frac{\varphi(-q)}{k^{1 / 4} \varphi\left(-q^{k}\right)},
$$

where $q=e^{-2 \pi \sqrt{n / k}}$, define $l_{k, n}$ by

$$
l_{k, n}:=\frac{\psi(-q)}{k^{1 / 4} q^{(k-1) / 8} \psi\left(-q^{k}\right)},
$$

where $q=e^{-\pi \sqrt{n / k}}$, define $l_{k, n}^{\prime}$ by

$$
l_{k, n}^{\prime}:=\frac{\psi(q)}{k^{1 / 4} q^{(k-1) / 8} \psi\left(q^{k}\right)},
$$

where $q=e^{-\pi \sqrt{n / k}}$

In $[7,8,10]$, several new modular equations for the theta functions were derived, some explicit relations and formulas for the parameterizations were offered, and some values of the parameterizations were determined. Moreover, in [9], some new modular equations of degrees 3 and 9 for the theta functions $\varphi$ and $\psi$ were derived in order to establish explicit relations and formulas for the parameterizations for $h_{k, n}, h_{k, n}^{\prime}, l_{k, n}$, and $l_{k, n}^{\prime}$ and show some applications of those modular equations to evaluations of the cubic continued fraction.

In this paper, we further derive some more modular equations of degrees 3 and 9 for the theta functions $\varphi$ and $\psi$ and present their concise proofs based on algebraic computations as in [9]. Furthermore, we find explicit relations and formulas for the corresponding parameterizations, evaluate some numerical values of $h_{k, n}, h_{k, n}^{\prime}, l_{k, n}$, and $l_{k, n}^{\prime}$ for some positive real numbers $k$ and $n$ by employing the relations and formulas established earlier, and evaluate some numerical values of the cubic continued fraction.

\section{Preliminary results}

In this section, we introduce fundamental theta function identities that will play key roles in deriving some modular equations. We also recall some useful explicit relations for the parameterizations of $h_{k, n}, l_{k, n}$, and $l_{k, n}^{\prime}$ for some positive real numbers $k$ and $n$. Let $k$ be the modulus as in (1.1). Set $x=k^{2}$ and also set

$$
k^{2}=x=1-\frac{\varphi^{4}(-q)}{\varphi^{4}(q)} .
$$

Then

$$
\varphi^{2}(q)={ }_{2} F_{1}\left(\frac{1}{2}, \frac{1}{2} ; 1 ; x\right)=: z,
$$


where

$$
q=e^{-y}:=\exp \left(-\pi \frac{{ }_{2} F_{1}\left(\frac{1}{2}, \frac{1}{2} ; 1 ; 1-x\right)}{{ }_{2} F_{1}\left(\frac{1}{2}, \frac{1}{2} ; 1 ; x\right)}\right)=\exp \left(-\pi \frac{K\left(k^{\prime}\right)}{K(k)}\right) .
$$

Lemma 2.1 ([1], Theorem 5.4.1). If $x, q$, and $z$ are related by (2.1), (2.2), and (2.3), then
(i) $\varphi(q)=\sqrt{z}$,
(ii) $\varphi(-q)=\sqrt{z}(1-x)^{1 / 4}$

Lemma 2.2 ([1], Theorem 5.4.2). If $x, q$, and $z$ are related by (2.1), (2.2), and (2.3), then
(i) $\psi(q)=\sqrt{\frac{1}{2} z}\left(\frac{x}{q}\right)^{1 / 8}$,
(ii) $\psi\left(q^{2}\right)=\frac{1}{2} \sqrt{z}\left(\frac{x}{q}\right)^{1 / 4}$

Lemma 2.3 ([2], Entry 1(ii), Chapter 20). For $|q|<1$, we have

$$
1+\frac{\psi\left(-q^{1 / 3}\right)}{q^{1 / 3} \psi\left(-q^{3}\right)}=\left(1+\frac{\psi^{4}(-q)}{q \psi^{4}\left(-q^{3}\right)}\right)^{1 / 3} .
$$

Lemma 2.4 ([2], Entry 5, Chapter 19). Let $\beta$ be the third degree and $m=\frac{z_{1}}{z_{3}}$. Then

(i) $\sqrt{\frac{\beta}{\alpha}}+\sqrt{\frac{1-\beta}{1-\alpha}}-\sqrt{\frac{\beta(1-\beta)}{\alpha(1-\alpha)}}=m^{2}$,

(ii) $\sqrt{\frac{\alpha}{\beta}}+\sqrt{\frac{1-\alpha}{1-\beta}}-\sqrt{\frac{\alpha(1-\alpha)}{\beta(1-\beta)}}=\left(\frac{3}{m}\right)^{2}$.

Lemma 2.5 ([2], Entry 3, Chapter 20). Let $\gamma$ be the ninth degree and $m=\frac{z_{1}}{z_{9}}$. Then

(i) $\left(\frac{\gamma}{\alpha}\right)^{1 / 8}+\left(\frac{1-\gamma}{1-\alpha}\right)^{1 / 8}-\left(\frac{\gamma(1-\gamma)}{\alpha(1-\alpha)}\right)^{1 / 8}=\sqrt{m}$,

(ii) $\left(\frac{\alpha}{\gamma}\right)^{1 / 8}+\left(\frac{1-\alpha}{1-\gamma}\right)^{1 / 8}-\left(\frac{\alpha(1-\alpha)}{\gamma(1-\gamma)}\right)^{1 / 8}=\frac{3}{\sqrt{m}}$.

Next two results will be useful in evaluating specific values of $h_{k, n}, h_{k, n}^{\prime}$, $l_{k, n}$, and $l_{k, n}^{\prime}$.

Lemma 2.6 ([8], Theorem 2.2). For any positive real number $k$,

$$
h_{k, 1}=1 \text {. }
$$

Lemma 2.7 ([10], Theorem 2.3). For any positive real number $k$,

$$
l_{k, 1}=1 \text {. }
$$


Lemma 2.8 ([9], Corollary 3.14). For every positive real number $n$, we have

$$
\left(l_{9, n}-l_{9, n}^{\prime}+\sqrt{3}\right)\left(\frac{1}{l_{9, n}}-\frac{1}{l_{9, n}^{\prime}}+\sqrt{3}\right)=1 .
$$

We recall that the cubic continued fraction $G(q)$ is defined by

$$
G(q):=\frac{q^{1 / 3}}{1}+\frac{q+q^{2}}{1}+\frac{q^{2}+q^{4}}{1}+\frac{q^{3}+q^{6}}{1}+\ldots=q^{1 / 3} \frac{\chi(-q)}{\chi^{3}\left(-q^{3}\right)},
$$

for $|q|<1$, where $\chi(q):=\left(-q ; q^{2}\right)_{\infty}$.

The following result exhibits some general formulas for the values of $G\left(e^{-\pi \sqrt{n}}\right)$ and $G\left(-e^{-\pi \sqrt{n}}\right)$ in terms of $l_{3, n}^{\prime}, l_{9, n}^{\prime}$, and $l_{9, n}$.

Lemma 2.9 ([10], Theorem 6.2). For any positive real number $n$, we have

(i) $G\left(e^{-\pi \sqrt{n}}\right)=\frac{1}{\sqrt{3} l_{9, n}^{\prime}-1}$,

(ii) $G^{3}\left(e^{-\pi \sqrt{n / 3}}\right)=\frac{1}{3 l_{3, n}^{\prime 4}-1}$

(iii) $G\left(-e^{-\pi \sqrt{n}}\right)=\frac{-1}{\sqrt{3} l_{9, n}+1}$.

Lemma 2.10 ([7], Lemma 6.3.6). We have

$$
G\left(e^{-2 \pi \sqrt{n}}\right)=-G\left(e^{-\pi \sqrt{n}}\right) G\left(-e^{-\pi \sqrt{n}}\right)
$$

for any positive real number $n$.

\section{Modular equations}

Note that Ramanujan's 23 eta function identities, which are certain types of modular equations, are given in [3]. In this section, we derive some modular equations of degrees 3 and 9 and present their proofs based on concise algebraic computations. In addition, we establish some explicit relations and formulas for $h_{k, n}, h_{k, n}^{\prime}, l_{k, n}$, and $l_{k, n}^{\prime}$ by employing these modular equations.

Theorem 3.1. If $P=\frac{\psi(q)}{q^{1 / 4} \psi\left(q^{3}\right)}$ and $Q=\frac{\psi\left(q^{2}\right)}{q^{1 / 2} \psi\left(q^{6}\right)}$, then

$$
P^{4}+\frac{9}{P^{4}}=\left(\frac{P}{Q}\right)^{4}+\left(\frac{Q}{P}\right)^{4}+8
$$

Proof. By Lemma 2.2,

$$
P=\sqrt{\frac{z_{1}}{z_{3}}}\left(\frac{\alpha}{\beta}\right)^{1 / 8} \text { and } Q=\sqrt{\frac{z_{1}}{z_{3}}}\left(\frac{\alpha}{\beta}\right)^{1 / 4},
$$

where $\beta$ has degree 3 over $\alpha$. Thus

$$
\frac{P^{2}}{Q}=\sqrt{\frac{z_{1}}{z_{3}}} \text { and } \quad \frac{Q}{P}=\left(\frac{\alpha}{\beta}\right)^{1 / 8}
$$


By Lemma 2.4,

$$
\left(\frac{P}{Q}\right)^{4}+\sqrt{\frac{1-\beta}{1-\alpha}}-\left(\frac{P}{Q}\right)^{4} \sqrt{\frac{1-\beta}{1-\alpha}}=\frac{P^{8}}{Q^{4}}
$$

and

$$
\left(\frac{Q}{P}\right)^{4}+\sqrt{\frac{1-\alpha}{1-\beta}}-\left(\frac{Q}{P}\right)^{4} \sqrt{\frac{1-\alpha}{1-\beta}}=\frac{9 Q^{4}}{P^{8}} .
$$

Combining and rearranging above two equations in terms of $P$ and $Q$, we deduce that

$$
\left(1-\frac{P^{4}}{Q^{4}}\right)\left(1-\frac{Q^{4}}{P^{4}}\right)=\left(P^{4}-1\right)\left(\frac{9}{P^{4}}-1\right),
$$

which is equivalent to (3.1). Hence we complete the proof.

Using the definition of $l_{k, n}^{\prime}$, we have the following:

Corollary 3.2. For every positive real number $n$, we have

$$
3\left(l_{3, n}^{\prime 4}+\frac{1}{l_{3, n}^{\prime 4}}\right)=\left(\frac{l_{3, n}^{\prime}}{l_{3,4 n}^{\prime}}\right)^{4}+\left(\frac{l_{3,4 n}^{\prime}}{l_{3, n}^{\prime}}\right)^{4}+8 .
$$

Proof. Letting $q=e^{-\pi \sqrt{n / 3}}$ in (1.6), we find that $P=3^{1 / 4} l_{3, n}^{\prime}$ and $Q=$ $3^{1 / 4} l_{3,4 n}^{\prime}$ in Theorem 3.1. Rewriting (3.1) in terms of $l_{3, n}^{\prime}$ and $l_{3,4 n}^{\prime}$, we complete the proof.

Theorem 3.3. If $P=\frac{\varphi(q)}{\varphi\left(q^{9}\right)}$ and $Q=\frac{\psi\left(q^{2}\right)}{q^{2} \psi\left(q^{18}\right)}$, then

$$
\sqrt{P Q}+\frac{3}{\sqrt{P Q}}=\sqrt{\frac{Q}{P}}+\sqrt{\frac{P}{Q}}+2 .
$$

Proof. By Lemma 2.1,

$$
P=\sqrt{\frac{z_{1}}{z_{9}}} \text { and } Q=\sqrt{\frac{z_{1}}{z_{9}}}\left(\frac{\alpha}{\gamma}\right)^{1 / 4},
$$

where $\gamma$ has degree 9 over $\alpha$. Thus

$$
\frac{Q}{P}=\left(\frac{\alpha}{\gamma}\right)^{1 / 4}
$$

By Lemma 2.5,

$$
\sqrt{\frac{P}{Q}}+\left(\frac{1-\gamma}{1-\alpha}\right)^{1 / 8}-\sqrt{\frac{P}{Q}}\left(\frac{1-\gamma}{1-\alpha}\right)^{1 / 8}=P
$$

and

$$
\sqrt{\frac{Q}{P}}+\left(\frac{1-\alpha}{1-\gamma}\right)^{1 / 8}-\sqrt{\frac{Q}{P}}\left(\frac{1-\alpha}{1-\gamma}\right)^{1 / 8}=\frac{3}{P}
$$


Combining and rearranging above two equations in terms of $P$ and $Q$, we deduce that

$$
\left(1-\sqrt{\frac{P}{Q}}\right)\left(1-\sqrt{\frac{Q}{P}}\right)=\left(P-\sqrt{\frac{P}{Q}}\right)\left(\frac{3}{P}-\sqrt{\frac{Q}{P}}\right),
$$

which is equivalent to (3.3). Hence we complete the proof.

Using the definitions of $h_{k, n}$ and $l_{k, n}^{\prime}$, we have the following:

Corollary 3.4. For every positive real number $n$, we have

$$
\sqrt{3}\left(\sqrt{h_{9, n} l_{9,4 n}^{\prime}}+\frac{1}{\sqrt{h_{9, n} l_{9,4 n}^{\prime}}}\right)=\sqrt{\frac{h_{9, n}}{l_{9,4 n}^{\prime}}}+\sqrt{\frac{l_{9,4 n}^{\prime}}{h_{9, n}}}+2 .
$$

Proof. Letting $q=e^{-\pi \sqrt{n / 9}}$ in (1.3) and (1.6), we find that $P=\sqrt{3} h_{9, n}$ and $Q=\sqrt{3} l_{9,4 n}^{\prime}$ in Theorem 3.3. Rewriting (3.3) in terms of $h_{9, n}$ and $l_{9,4 n}^{\prime}$, we complete the proof.

Theorem 3.5. If $P=\frac{\psi(-q)}{q \psi\left(-q^{9}\right)}$ and $Q=\frac{\psi\left(-q^{3}\right)}{q^{3} \psi\left(-q^{27}\right)}$, then

$$
\left(P+3+\frac{3}{P}\right)\left(Q+3+\frac{3}{Q}\right)=\left(\frac{Q}{P}\right)^{2}
$$

Proof. For simplicity, let $A=q \psi\left(-q^{9}\right)$ and $B=q^{3} \psi\left(-q^{27}\right)$. Then $\psi(-q)=A P$ and $\psi\left(-q^{3}\right)=B Q$. Thus by Lemma 2.3 ,

$$
P+1=\left(\frac{q B^{4} Q^{4}}{A^{4}}+1\right)^{1 / 3} \text { and } Q+1=\left(\frac{A^{4}}{q B^{4}}+1\right)^{1 / 3} .
$$

Combining and rewriting above two equations in terms of $P$ and $Q$, we deduce that

$$
\left((P+1)^{3}-1\right)\left((Q+1)^{3}-1\right)=Q^{4},
$$

which is equivalent to (3.3). Hence we complete the proof.

Using the definition of $l_{k, n}$, we have the following:

Corollary 3.6. For every positive real number $n$, we have

$$
3\left(l_{9, n}+\sqrt{3}+\frac{1}{l_{9, n}}\right)\left(l_{9,9 n}+\sqrt{3}+\frac{1}{l_{9,9 n}}\right)=\left(\frac{l_{9,9 n}}{l_{9, n}}\right)^{2} .
$$

Proof. Letting $q=e^{-\pi \sqrt{n / 9}}$ in (1.5), we find that $P=\sqrt{3} l_{9, n}$ and $Q=\sqrt{3} l_{9,9 n}$ in Theorem 3.5. Rewriting (3.5) in terms of $l_{9, n}$ and $l_{9,9 n}$, we complete the proof. 


\section{Evaluations of $l_{k, n}$ and $l_{k, n}^{\prime}$}

In this section, we evaluate specific values of $l_{k, n}$ and $l_{k, n}^{\prime}$ for some positive real numbers $k$ and $n$ by using the explicit relations and formulas established in Section 3.

The following results exhibit a general method for evaluating the values of $l_{3,4^{n}}^{\prime}$ for all positive integers $n$. We show the case when $n=1, n=2$, and $n=3$.

Theorem 4.1. We have

(i) $l_{3,4}^{\prime}=\frac{1+\sqrt{3}}{\sqrt{2}}$,

(ii) $l_{3,16}^{\prime}=1+\sqrt{3}+\sqrt{2+\sqrt{3}}$,

(iii) $l_{3,64}^{\prime}=\left(\frac{3 a^{8}-8 a^{4}+3+\sqrt{3}\left(a^{4}-1\right) \sqrt{\left(3 a^{4}-1\right)\left(a^{4}-3\right)}}{2}\right)^{1 / 4}$,

where

$$
a=1+\sqrt{3}+\sqrt{2+\sqrt{3}} .
$$

Proof. For (i), letting $n=1$ in $(3.2)$ and putting the value of $l_{3,1}^{\prime}=(2+\sqrt{3})^{1 / 4}$ from Theorem 4.3(i) in [9], we find that

$$
(2-\sqrt{3}) l_{3,4}^{\prime 8}-4 l_{3,4}^{\prime 4}+2+\sqrt{3}=0 .
$$

Solving for $l_{3,4}^{\prime}$ and using the fact that $l_{3,4}^{\prime}$ has a real value greater than 1 , we complete the proof.

For (ii), letting $n=4$ in (3.2) and putting the value of $l_{3,4}^{\prime}$ from the previous result of (i), we find that

$$
l_{3,16}^{\prime 8}-34(7+4 \sqrt{3}) l_{3,16}^{\prime 4}+97+56 \sqrt{3}=0 .
$$

Solving for $l_{3,16}^{\prime}$ and using the fact that $l_{3,16}^{\prime}$ has a real value greater than 1 , we complete the proof.

For (iii), letting $n=16$ in (3.2) and putting the value of $l_{3,16}^{\prime}$ from the previous result of (ii), we find that

$$
l_{3,64}^{\prime 8}-\left(3 a^{8}-8 a^{4}+3\right) l_{3,64}^{\prime 4}+a^{8}=0,
$$

where

$$
a=1+\sqrt{3}+\sqrt{2+\sqrt{3}} .
$$

Solving for $l_{3,64}^{\prime}$ and using the fact that $l_{3,64}^{\prime}$ has a real value greater than 1 , we complete the proof.

See Theorem 4.10(ix) in [10] for an alternative proof for Theorem 4.1(i). By repeating the same argument as in the proof of Theorem 4.1, we can evaluate the values of $l_{3,4^{n}}^{\prime}$ for $n=4,5,6, \ldots$

Theorem 4.2. We have 
(i) $l_{9,4}^{\prime}=1+\sqrt{3}+\sqrt{3+2 \sqrt{3}}$,

(ii) $l_{9,36}^{\prime}=\frac{\sqrt{3} a^{2}-2 a+\sqrt{3}+2 \sqrt{\sqrt{3} a\left(a^{2}-\sqrt{3} a+1\right)}}{(\sqrt{3} a-1)^{2}}$,

where

$$
a=2-\sqrt{3}+(38-22 \sqrt{3})^{1 / 3}+\frac{2(5-3 \sqrt{3})}{(38-22 \sqrt{3})^{1 / 3}},
$$

Proof. For (i), letting $n=1$ in (3.4) and using $h_{9,1}=1$ from Lemma 2.7, we find that

$$
l_{9,4}^{\prime 2}-2(1+\sqrt{3}) l_{9,4}^{\prime}+1=0 .
$$

Solving for $l_{9,4}^{\prime}$ and noting the fact that $l_{9,4}^{\prime}>1$, we complete the proof.

For (ii), let $n=9$ in (3.4), then we have

$$
\left(\sqrt{3} h_{9,9}-1\right) l_{9,36}^{\prime}-2 \sqrt{h_{9,9} l_{9,36}^{\prime}}-h_{9,9}+\sqrt{3}=0,
$$

where

$$
h_{9,9}=2-\sqrt{3}+(38-22 \sqrt{3})^{1 / 3}+\frac{2(5-3 \sqrt{3})}{(38-22 \sqrt{3})^{1 / 3}}
$$

from Theorem 4.2(ii) in [9]. Now solving for $l_{9,36}^{\prime}$ and using the fact that $l_{9,36}^{\prime}>1$, we complete the proof.

See Theorem 4.3(iii) in [9] for an alternative proof for Theorem 4.2(i).

Corollary 4.3. Let $a$ be as in Theorem 4.2(ii). Then we have

$$
l_{9,36}=\frac{1}{2}\left(b-\sqrt{3}+\sqrt{\frac{\sqrt{3}\left(b^{2}+1\right)(b-\sqrt{3})}{\sqrt{3} b-1}}\right),
$$

where

$$
b=\frac{\sqrt{3} a^{2}-2 a+\sqrt{3}+2 \sqrt{\sqrt{3} a\left(a^{2}-\sqrt{3} a+1\right)}}{(\sqrt{3} a-1)^{2}} .
$$

Proof. Let $n=36$ in Lemma 2.8 and $b=l_{9,36}^{\prime}$. Then we find that

$$
(\sqrt{3} b-1) l_{9,36}^{2}-\left(\sqrt{3} b^{2}-4 b+\sqrt{3}\right) l_{9,36}-b^{2}+\sqrt{3} b=0 .
$$

Putting the value of $b$ from Theorem 4.2(ii), solving for $l_{9,36}$, and using the fact that $l_{9,36}>1$, we complete the proof.

The following results exhibit a general method for evaluating the values of $l_{9,9^{n}}$ for all positive integers $n$. We show the case when $n=1$ and $n=2$.

Theorem 4.4. We have

(i) $l_{9,9}=2+\sqrt{3}+(38+22 \sqrt{3})^{1 / 3}+\frac{2(5+3 \sqrt{3})}{(38+22 \sqrt{3})^{1 / 3}}$,

(ii) $l_{9,81}=\left(\left(a^{2}+1\right)(a+\sqrt{3})\right)^{2 / 3} b^{1 / 3}+\left(\left(a^{2}+1\right)(a+\sqrt{3})\right)^{1 / 3} b^{2 / 3}+b$, 
where

$$
\begin{gathered}
a=2+\sqrt{3}+(38+22 \sqrt{3})^{1 / 3}+\frac{2(5+3 \sqrt{3})}{(38+22 \sqrt{3})^{1 / 3}}, \\
b=a\left(a^{2}+\sqrt{3} a+1\right) .
\end{gathered}
$$

Proof. For (i), letting $n=1$ in (3.6) and using $l_{9,1}=1$ from Lemma 2.7, we find that

$$
l_{9,9}^{3}-3(2+\sqrt{3}) l_{9,9}^{2}-3(3+2 \sqrt{3}) l_{9,9}-3(2+\sqrt{3})=0 .
$$

Solving for $l_{9,9}$ and noting the fact that $l_{9,9}$ is real valued, we complete the proof.

For (ii), let $n=9$ in (3.6), then we have

$$
3\left(l_{9,9}+\sqrt{3}+\frac{1}{l_{9,9}}\right)\left(l_{9,81}+\sqrt{3}+\frac{1}{l_{9,81}}\right)=\left(\frac{l_{9,81}}{l_{9,9}}\right)^{2} .
$$

Putting the value of $l_{9,9}$ from the previous result of (i), solving for $l_{9,81}$, and using the fact that $l_{9,81}$ is real valued, we complete the proof.

See Theorem 4.4(ii) in [9] for an alternative proof for Theorem 4.4(i). By repeating the same argument as in the proof of Theorem 4.4, we can evaluate the values of $l_{9,9^{n}}$ for $n=3,4,5, \ldots$.

Corollary 4.5. Let $a$ and $b$ be as in Theorem 4.4(ii). Then we have

$$
l_{9,81}^{\prime}=\frac{1}{2}\left(c+\sqrt{3}+\sqrt{\frac{\sqrt{3}\left(c^{2}+1\right)(c+\sqrt{3})}{\sqrt{3} c+1}}\right),
$$

where

$$
c=\left(\left(a^{2}+1\right)(a+\sqrt{3})\right)^{2 / 3} b^{1 / 3}+\left(\left(a^{2}+1\right)(a+\sqrt{3})\right)^{1 / 3} b^{2 / 3}+b .
$$

Proof. The result follows directly from Lemma 2.8 and Theorem 4.4(ii).

\section{Evaluations of $G(q)$}

We now turn to an application of some numerical values of $l_{k, n}$ and $l_{k, n}^{\prime}$ for some positive real numbers $k$ and $n$ to evaluations of the cubic continued fraction. In [7], the values of $G\left(e^{-\pi / \sqrt{3}}\right), G\left(-e^{-\pi / \sqrt{3}}\right)$, and $G\left(e^{-2 \pi / \sqrt{3}}\right)$ were evaluated. In particular, $G\left(e^{-2 \pi / \sqrt{3}}\right)$ was evaluated by multiplying both $-G\left(e^{-\pi / \sqrt{3}}\right)$ and $G\left(-e^{-\pi / \sqrt{3}}\right)$ as in Lemma 2.9. In this section, we evaluate $G\left(e^{-2 \pi / \sqrt{3}}\right)$ by putting the value of $l_{3,4}^{\prime}$ in Lemma 2.9(ii). Moreover, we evaluate the numerical values of $G\left(e^{-4 \pi / \sqrt{3}}\right), G\left(e^{-8 \pi / \sqrt{3}}\right), G\left(-e^{-2 \pi / \sqrt{3}}\right)$, and $G\left(-e^{-4 \pi / \sqrt{3}}\right)$.

Theorem 5.1. We have

(i) $G\left(e^{-2 \pi / \sqrt{3}}\right)=\frac{1}{2}(-5+3 \sqrt{3})^{1 / 3}$, 
(ii) $G\left(e^{-4 \pi / \sqrt{3}}\right)=\frac{1}{2}(7+6 \sqrt{3}-9 \sqrt{2+\sqrt{3}})^{1 / 3}$,

(iii) $G\left(e^{-8 \pi / \sqrt{3}}\right)=\frac{1}{2^{4 / 3}}\left(3 a^{4}-7-3 \sqrt{3}\left(a^{4}-1\right) \sqrt{\frac{a^{4}-3}{3 a^{4}-1}}\right)^{1 / 3}$, where

$$
a=1+\sqrt{3}+\sqrt{2+\sqrt{3}}
$$

Proof. For (i), letting $n=4$ in Lemma 2.9(ii) and putting the value of $l_{3,4}^{\prime}$ from Theorem 4.1(i), we complete the proof.

For (ii), letting $n=16$ in Lemma 2.9 (ii) and putting the value of $l_{3,16}^{\prime}$ from Theorem 4.1(ii), we complete the proof.

For (iii), letting $n=64$ in Lemma 2.9 (ii) and putting the value of $l_{3,64}^{\prime}$ from Theorem 4.1(iii), we complete the proof.

Corollary 5.2. We have

$$
\begin{aligned}
\text { (i) } G\left(-e^{-2 \pi / \sqrt{3}}\right) & =-\left(\frac{1}{2}(5+3 \sqrt{3})(7+6 \sqrt{3}-9 \sqrt{2+\sqrt{3}})\right)^{1 / 3}, \\
\text { (ii) } G\left(-e^{-4 \pi / \sqrt{3}}\right) & =-\left(\frac{\left(3 a^{4}-7\right) \sqrt{3 a^{4}-1}-3 \sqrt{3}\left(a^{4}-1\right) \sqrt{a^{4}-3}}{2(7+6 \sqrt{3}-9 \sqrt{2+\sqrt{3}}) \sqrt{3 a^{4}-1}}\right)^{1 / 3},
\end{aligned}
$$

where

$$
a=1+\sqrt{3}+\sqrt{2+\sqrt{3}} .
$$

Proof. Parts (i) and (ii) follow directly from Lemma 2.10 and Theorem 5.1.

Note that the numerical values of $G\left(e^{-\pi}\right), G\left(e^{-2 \pi}\right)$, and $G\left(-e^{-\pi}\right)$ were evaluated in [4]. Note also that the numerical values of $G\left(e^{-\pi}\right), G\left(e^{-2 \pi}\right)$, $G\left(e^{-3 \pi}\right), G\left(-e^{-2 \pi}\right)$, and $G\left(-e^{-3 \pi}\right)$ were evaluated in [9]. Hence the numerical values of $G\left(e^{-\pi}\right)$ and $G\left(e^{-2 \pi}\right)$ were given in both [4] and [9], but they were evaluated by different proofs. We close this section by evaluating the numerical values of $G\left(e^{-6 \pi}\right), G\left(e^{-9 \pi}\right), G\left(e^{-12 \pi}\right), G\left(e^{-18 \pi}\right), G\left(-e^{-6 \pi}\right)$, and $G\left(-e^{-9 \pi}\right)$.

Theorem 5.3. Let $a$ and $b$ be as in Corollary 4.3. Then we have

(i) $G\left(e^{-6 \pi}\right)=\frac{(\sqrt{3} a-1)^{2}}{2\left(1+3^{3 / 4} \sqrt{a\left(a^{2}-\sqrt{3} a+1\right)}\right)}$,

(ii) $G\left(-e^{-6 \pi}\right)=-\frac{1}{4}\left((\sqrt{3} b-1)^{2}-3^{3 / 4} \sqrt{\left(b^{2}+1\right)(b-\sqrt{3})(\sqrt{3} b-1)}\right)$.

Proof. Part (i) follows directly from Lemma 2.9(i) and Theorem 4.2(ii). Part (ii) follows directly from Lemma 2.9(iii) and Corollary 4.3. 
Corollary 5.4. Let $a$ and $b$ be as in Corollary 4.3. Then we have

$$
G\left(e^{-12 \pi}\right)=\frac{(\sqrt{3} a-1)^{2}\left((\sqrt{3} b-1)^{2}-3^{3 / 4} \sqrt{\left(b^{2}+1\right)(b-\sqrt{3})(\sqrt{3} b-1)}\right)}{8\left(1+3^{3 / 4} \sqrt{a\left(a^{2}-\sqrt{3} a+1\right)}\right)} .
$$

Proof. The result follows directly from Lemma 2.10 and Theorem 5.3.

Theorem 5.5. Let $a$ and $b$ be as in Theorem 4.4(ii). Then we have

where

$$
G\left(-e^{-9 \pi}\right)=-\frac{1}{1+\sqrt{3} c}
$$

$$
c=\left(\left(a^{2}+1\right)(a+\sqrt{3})\right)^{2 / 3} b^{1 / 3}+\left(\left(a^{2}+1\right)(a+\sqrt{3})\right)^{1 / 3} b^{2 / 3}+b .
$$

Proof. Letting $n=81$ in Lemma 2.9 (iii) and putting the value of $l_{9,81}$ from Theorem 4.4(ii), we complete the proof.

Theorem 5.6. Let $c$ be as in Corollary 4.5. Then we have

$$
G\left(e^{-9 \pi}\right)=\frac{1}{4}\left(3^{3 / 4} \sqrt{\left(c^{2}+1\right)(c+\sqrt{3})(\sqrt{3} c+1)}-(\sqrt{3} c+1)^{2}\right) .
$$

Proof. Letting $n=81$ in Lemma 2.9(i) and putting the value of $l_{9,81}^{\prime}$ from Corollary 4.5, we complete the proof.

Corollary 5.7. Let $c$ be as in Corollary 4.5. Then we have

$$
G\left(e^{-18 \pi}\right)=\frac{3^{3 / 4} \sqrt{\left(c^{2}+1\right)(c+\sqrt{3})(\sqrt{3} c+1)}-(\sqrt{3} c+1)^{2}}{4(\sqrt{3} c+1)} .
$$

Proof. The result is an immediate consequence of Lemma 2.10 and Theorems 5.5 and 5.6 .

\section{References}

[1] B. C. Berndt, Number Theory in the Spirit of Ramanujan, American Mathematical Society, 2006.

[2] — Ramanujan's Notebooks, Part III, Springer-Verlag, New York, 1991.

[3] — Ramanujan's Notebooks, Part IV, Springer-Verlag, New York, 1994.

[4] H. H. Chan, On Ramanujan's cubic continued fraction, Acta Arith. 73 (1995), no. 4, $343-355$.

[5] R. A. Rankin, Modular Forms and Functions, Cambridge University Press, Cambridge, 1977.

[6] B. Schoeneberg, Elliptic Modular Functions, Springer-Verlag, New York, 1974.

[7] J. Yi, The Construction and Applications of Modular Equations, Ph. D. Thesis, University of Illinois at Urbana-Champaign, 2001.

[8] — Theta-function identities and the explicit formulas for theta-function and their applications, J. Math. Anal. Appl. 292 (2004), no. 2, 381-400. 
[9] J. Yi, M. G. Cho, J. H. Kim, S. H. Lee, J. M. Yu, and D. H. Paek, On some modular equations and their applications I, Bull. Korean Math. Soc. 50 (2013), no. 3, 761-776.

[10] J. Yi, Y. Lee, and D. H. Paek, The explicit formulas and evaluations of Ramanujan's theta-function $\psi$, J. Math. Anal. Appl. 321 (2006), no. 1, 157-181.

Dae Hyun Paek

Department of Mathematics Education

Busan National University of Education

BUSAN 611-736, KOREA

E-mail address: paek@bnue.ac.kr

JINHEE Yi

Department of Mathematics And Computer Science

Korea Science Academy of KAIST

BusAn 614-822, KorEA

E-mail address: jhyi100@kaist.ac.kr 